It has shown that the HCAs who participated found that their knowledge and confidence around the techniques increased. The impact of this service development however was difficult to measure due to poor uptake and evaluation of the educative strategies employed.

This service development has demonstrated that the education of HCAs has the potential to improve the rehabilitation of palliative care patients but that this needs to be delivered in a way that 'fits around' the daily work of HCAs and has to be recognised as an important part in the culture of the hospice.

Those who participated in the service development evaluated it positively, feeling that they were in the position to carry out the techniques and practice with the patients. A further outcome of the development is to consider different ways to provide the classroom teaching and clinical facilitation within the organisation to allow greater participation from staff

\section{P71 A SERVICE DEVELOPMENT TO EDUCATE HEALTHCARE ASSISTANTS ABOUT PHYSICAL REHABILITATION IN AN IN-PATIENT HOSPICE UNIT}

Rachel Atkinson, Sally Jennings LOROS Hospice Leicestershire England

10.1136/bmjspcare-2011-000105.71

Rehabilitation is a specialty in its own right, with a powerful emphasis on inter-professional working to help patients improve their independence. Due to medical advances people with life limiting illnesses are living longer and even though they will not be cured rehabilitation has a vital role in improving their lives over the time they have ahead. This concept may seem strange in the context of palliative care but the goals of rehabilitation and palliative care are the same in helping a person to become as active and independent as possible.

The aim of this service development is to educate Healthcare Assistants (HCAs) to promote a physical rehabilitation approach in a 31 bedded in-patient hospice unit that focuses on independence.

A series of education and practice interventions were developed. They were implemented through group tutorials, display boards and clinical facilitation, with an evaluation of all of these strategies. 